\title{
System Accuracy Evaluation for Blood Glucose Measurements Should Be Performed in Accordance with ISO 15197:2003 and CLSI-Approved Guideline EP7-A2
}

\author{
Rolf Hinzmann, M.D., Ph.D., and Cam-Tuan Tran, M.D.
}

Dear Editor:

W ARNER ET AL. ${ }^{1}$ RECENTLY reported potential risks to patient welfare with the use of bedside rather than laboratory blood glucose $(\mathrm{bG})$ measurement on bG meters such as the Accu-Chek ${ }^{\circledR}$ Performa (Roche Diagnostics, Mannheim, Germany). Warner et al. ${ }^{1}$ are claiming that this bG meter potentially fails to diagnose hypoglycemia and might lead to overtreatment of hyperglycemia in patients with high blood maltose concentrations and high hematocrit values. Our review of their methods identified several key discrepancies that may help to explain their conclusions.

First, the manufacturer's recommended comparison method of the Accu-Chek Performa bG meter is the hexokinase method with deproteinization of capillary whole blood samples. This hexokinase method is directly calibrated to the reference measurement procedure isotope dilution gas chromatography/ mass spectrometry (ID-GC/MS), which is a reference procedure recommended by the Joint Committee for Traceability in Laboratory Medicine (JCTLM) ${ }^{2}$ (JCTLM DB identification number: C3RMMP19, isotope dilution mass spectrometry method for glucose in whole blood). The Vitros 5,1 FS (Ortho Clinical Diagnostics, Raritan, NJ) glucose method against which Warner et al. ${ }^{1}$ did compare the Accu-Chek Performa is a glucose oxidase-peroxidase method using plasma. Use of the Vitros 5,1 FS as a comparison method is not in accordance with the recommendation of the International Organization for Standardization (ISO) 15197:2003, ${ }^{3}$ which requires that bG meters are being evaluated by the comparison method recommended by the manufacturer or by another validated measurement procedure that has been shown to produce equivalent results. The Vitros 5,1 FS glucose method is not the comparison method that has been recommended by Roche. In addition, the glucose measurement on a clinical chemical analyzer is associated with a measurement error. As we know from external quality assessment schemes (EQAS), the variation of glucose measurement values between different clinical chemical analyzers can be significant. An example is that the (German) Referenzinstitut für Bioanalytik publishes EQAS results with open access to the public on its website. A sample from a recent EQAS (May 2, 2011) with a glucose reference value (determined by ID-GC/MS) of $76.6 \mathrm{mg} / \mathrm{dL}$ had an inter-laboratory coefficient of variation of $5.95 \% .{ }^{4}$ The authors would have had to run the Vitros 5,1 FS glucose method and the method recommended by Roche side by side to prove equivalence. It is nowhere indicated in their publication that they did so.

Furthermore, ISO 15197:2003 requires that the system accuracy evaluation shall be performed with at least 100 fresh capillary blood samples, each with sufficient volume to be measured by two different bG meters and at least in duplicate by the manufacturer's recommended measurement procedure. The study from Warner et al. ${ }^{1}$ provides information about only 40 samples with no further details supporting the conclusions made by the authors.

Second, data for maltose mentioned in the publication of Warner et al. ${ }^{1}$ are based on a pharmacodynamic modeling calculation published by Schleis, ${ }^{5}$ which yielded a theoretical maximum maltose concentration of $2,650 \mathrm{mg} / \mathrm{dL}$ (77 mmol/ L). The calculation is based on two assumptions: (1) that no maltose is converted during the infusion process and (2) that the infusion is essentially instantaneous. To date, the concentration of 2,650 mg/dL maltose has never been observed in real-life patients. Assuming a maltose concentration of $600 \mathrm{mg} / \mathrm{dL}(17.5 \mathrm{mmol} / \mathrm{L})$, which is the maximum that has been observed in an analysis of six real-life infusion experiments by Schleis, ${ }^{5}$ would change the true glucose value of $39.6 \mathrm{mg} / \mathrm{dL}(2.2 \mathrm{mmol} / \mathrm{L})$ to $54.0 \mathrm{mg} / \mathrm{dL}(3.0 \mathrm{mmol} / \mathrm{L})$ as shown by Warner et al. ${ }^{6}$ This would not lead to inappropriate treatment.

Third, according to the guideline of the Clinical and Laboratory Standards Institute (CLSI) for interference testing in clinical chemistry, ${ }^{7}$ it is necessary for the assessment of an interferent such as hematocrit to compare samples with the same glucose concentration and different hematocrit values with each other only on the same instrument. This procedure isolates the effect of the interferent, while any comparison to a different instrument results in a deviation that is not only due to the interferent but also partly due to the measurement error of the different instrument. The hematocrit figures in the publication of Warner et al. ${ }^{1}$ showed glucose concentrations at different hematocrit concentrations that were measured with the Accu-Chek Performa and then compared with the corresponding values measured with the Vitros 5,1 FS.

Diabetes Care, Roche Diagnostics GmbH, Mannheim, Germany. 
If Warner et al. ${ }^{1}$ had followed the CLSI guideline for interference testing, the observed deviation would have been much smaller and would then lead to a different assessment.

In summary, in our opinion it can therefore be stated that the conclusions in Warner at al. ${ }^{1}$ regarding the maltose interference and the putative evidence of a positive bias at higher hematocrit values with the Accu-Chek Performa are not supported because of the fact that the experiments have not been performed according to the ISO 15197:2003 standard $^{3}$ and the CLSI guideline for interference testing. ${ }^{7}$

\section{References}

1. Warner JV, Wu JY, Buckingham N, McLeod DS, Mottram B, Carter AC: Can one point-of-care glucose meter be used for all pediatric and adult hospital patients? Evaluation of three meters, including recently modified test strips. Diabetes Technol Ther 2011;13:55-62.

2. Reference Measurement Methods/Procedures. www.bipm.org/ jctlm/viewResults.do?type $=$ isRMP\&searchString $=$ glucose $\&$ searchStringIUPAC $=$ \&searchStringMixed $=$ \&analyteCategory $=$ Metabolites + and + substrates\&matrixCategory $=$ Whole + blood\&sortBy $=$ Analyte_Name\&status $=$ P\&id = C3RMMP19* $6^{*} 10 \& x=39 \& y=9$ (accessed December 14, 2011).

3. ISO 15297:2003. In Vitro Diagnostic Test Systems-Requirements for Blood-Glucose Monitoring Systems for Self-Testing in Managing Diabetes Mellitus. www.iso.org/iso/iso_catalogue/ catalogue_tc/catalogue_detail.htm?csnumber $=26309$ (accessed December 14, 2011).

4. Youdendiagramme KS4/11. www.dgkl-rfb.de/4daction/g_send _pdfGET /Youdenplots_KS4/11.pdf?rvID =KS114\&year = 2011\& type $=$ plot\&split $=1 \&$ lang $=\mathrm{d}$ (accessed December 14, 2011).

5. Schleis T: Interference of maltose, icodextrin, galactose, or xylose with some blood glucose monitoring systems. Pharmacotherapy 2007;27:1313-1321.

6. Warner JV, Wu JY, Buckingham N, McLeod DSA, Carter AC: P91 Evaluation of Hemocue, Accu-Chek and Optium blood glucose meters for point-of-care use in paediatric and adult hospital patients [sub-article 114]. Clin Biochem Rev 2009;30:S7-S47.

7. Clinical and Laboratory Standards Institute: Interference Testing in Clinical Chemistry: Approved Guidelines, $2^{\text {nd }}$ ed. CLSI document EP7-A2, Clinical and Laboratory Standards Institute, Wayne, PA, 2005.

Address correspondence to: Rolf Hinzmann, M.D., Ph.D. Diabetes Care

Roche Diagnostics $\mathrm{GmbH}$ Sandhofer Straße 116 68305 Mannheim, Germany

E-mail: rolf.hinzmann@roche.com

Website: www.roche.com 